https://doi.org/10.22519/22157360.703

\title{
Selección de personal por competencias en las empresas de servicios
}

\section{Personnel selection for competency on service companies}

\author{
Mauricio Pérez Perales ${ }^{1}$
}

\section{Resumen}

El presente artículo tuvo como objetivo analizar el proceso de selección del personal por competencias en las empresas del sector servicios. Debido a que la calidad del servicio que se les brinda a los clientes es el factor que constituye el elemento diferenciador y que da un elevado valor agregado a la organización frente a otras en el mercado, el servir al cliente por medio de competencias laborales debe ser comprendido como una estrategia en la que toda la empresa debe de estar enfocada. Resulta indispensable entonces, contar con personal idóneo y capacitado para satisfacer las necesidades y expectativas de los grupos de interés relacionados con la organización.

Palabras clave: Competencia laboral; Gestión humana por competencias; selección de personal; técnicas de selección; servicio al cliente.

\footnotetext{
${ }^{1}$ Administrador de Empresas. Universidad Los Libertadores maoperez.333@hotmail.com

AGLALA ISNN 2215-7360

2014; 5 (1): 107-125
} 
CÓDIGO JEL: M12

Fecha de recepción: Julio de 2014 / Fecha de aceptación en forma revisada: Septiembre 2014

\begin{abstract}
Objective of present paper was to analyze the process of selection of personnel competence in companies in the services sector. Because the quality of service they provide to clients is the factor that is the differentiator and gives a high value to the organization from others in the market, serving the customer through job skills should be understood as a strategy in which the whole company must be focused. It is essential then, have qualified and trained staff to meet the needs and expectations of stakeholders related to the organization.
\end{abstract}

Keywords: Labor competition; Human skill management; personnel selection; selection techniques; customer service.

\title{
Introducción
}

Actualmente, las organizaciones empresariales evolucionan con gran rapidez, el cual se caracteriza por profundos y dramáticos cambios que amenazan la existencia de toda empresa, obligándola a responder y adaptarse de manera dinámica a las nuevas realidades. La actual situación del mercado exige que las empresas estén un paso adelante tanto ante las necesidades de los clientes y usuarios, como de sus propias necesidades internas.

Las empresas, sin importar su tamaño o razón social, han comprendido que el capital monetario, financiero o el tecnológico no son suficientes para lograr una ventaja competitiva AGLALA ISNN 2215-7360

2014; 5 (1): 107-125 
frente a las demás empresas del mercado. Para esto requieren contar con un capital intelectual idóneo, es decir con personal calificado y competente que responda rápidamente al cambio y sea capaz de satisfacer las necesidades del mercado, permitiéndole así alcanzar sus objetivos.

Partiendo de lo anterior, puede observarse la gran importancia que el recurso humano tiene dentro de una organización, y que todos los procesos llevados a cabo para su selección y reclutamiento son parte fundamental para lograr el crecimiento y desarrollo de la organización; pues tienen como principal objeto seleccionar personas con habilidades, motivación y satisfacción suficientes para alcanzar los objetivos organizacionales.

La realización de esta investigación permitió conocer con mayor detalle la selección de personal por competencias en las empresas de servicios.

El trabajo realizado se enmarca dentro de la línea de innovación y emprendimiento, ya que se busca mejorar la calidad en el proceso de selección del personal que formará parte de las organizaciones. El tema articulador corresponde a la gestión humana por competencias, el criterio orientador al proceso de selección del personal.

El método de estudio es cualitativo y el tipo exploratorio y las fuentes de recolección de la información fueron las secundarias, ya que se utilizaron en la consulta libros, revistas y base de datos y la técnica que se desarrolló fue el análisis documental.

\section{La selección de personal por competencias}

La palabra competencia proviene del verbo en latín competere y según la Real Academia de la Lengua (2014), se derivan dos acepciones que son, en primer lugar, la palabra competir, que se refiere a la rivalidad entre dos o más personas sobre una situación determinada o entre empresas ante un mercado al cual ofrecen productos o servicios y, en segundo lugar, el vocablo AGLALA ISNN 2215-7360

2014; 5 (1): 107-125 
competente, que tiene que ver con habilidad y ser idóneo para una tarea. Ahora, esta última es la que apunta esta investigación.

La competencia se puede enfocar desde la perspectiva de Pereda y Berrocal (2011), quienes hablan sobre el progreso del enfoque del rasgo al enfoque del rasgo y de competencias. En el primero, la Dirección de Personal entabla predicciones sobre lo que los trabajadores rendirán, a través de modelos psicológicos que existen en torno a los rasgos. Debido a ello, se establecen qué tipo de rasgos psicológicos deben de contar los individuos que ostentarán un cargo y que a la vez respondan con éxito al mismo. Los candidatos son sujeto a evaluación a través de pruebas o entrevistas psicológicas, test que miden las aptitudes, la personalidad y los conocimientos. El segundo, se centra en el comportamiento de los individuos eficaces en su área laboral, el cual lleva a definir el puesto por medio de las conductas observables. Por lo tanto, estos autores definen la competencia como al conjunto de conductas que son observables y que se encuentran relacionados con un desenvolvimiento bueno o excelente en una determinada labor y en una organización concreta.

A las personas se les demanda mayores exigencias en su rendimiento y que vayan de la mano con la necesidad de incrementar las habilidades por medio de procesos de aprendizaje, capacitación y desarrollo permanente. Por medio de la alta competitividad, se hace cada vez más difícil que las empresas logren desarrollar ventajas competitivas reales. Blanco (2007), expresa que el enfoque de competencias valora el estudio del comportamiento sobre la inteligencia, siendo esta perspectiva visionada desde el punto de vista conductista. Sin embargo, el adoptar el enfoque de competencias no quiere decir que se tenga que dejar de lado el enfoque de los rasgos, sino que el nivel de inteligencia y las aptitudes de un individuo, acompañado de sus rasgos de personalidad, darán como consecuencia una mejor o menor facilidad al momento de desempeñar AGLALA ISNN 2215-7360

2014; 5 (1): 107-125 
una actividad, así como su potencialidad para aprender a realizar otras cosas. La norma actual es ofrecer productos y servicios de alta calidad con bajo costo, por eso las organizaciones ven en su personal la fuente de diferenciación ante la competencia.

Montoya (2010) expresa que cada competencia es comprendida como la integración de tres tipos de saberes, a saber: conceptual (saber), procedimental (saber hacer) y actitudinal (ser).

En lo concerniente al saber, se incluyen los conocimientos técnicos y de gestión. Lo del saber hacer, abarca las habilidades innatas del aprendizaje y la experiencia. Las que tienen que ver con el ser, son las aptitudes personales, comportamientos, actitudes, personalidad y los valores.

Helleriegel en el año 2009 expuso que una competencia se presenta a través de la combinación de conocimientos, conductas, habilidades y actitudes que favorecen a que la persona sea efectiva. Además, es la capacidad de dar movimiento y de dar aplicabilidad de manera correcta en el área laboral recursos propios del trabajador, como son habilidades, conocimientos, actitudes y recursos del entorno para dar a luz resultados previamente definidos y satisfactorios. Ahora, para que exista un buen desempeño y oportuno en diversos contextos se necesita ser flexible y tener capacidad de adaptación.

Este mismo autor divide las competencias para las organizaciones a través de las competencias conductuales, que son el conjunto de habilidades y comportamientos que dan un desempeño destacado en el trabajo y que se pueden hacer visibles por medio de la pro actividad, la flexibilidad, la innovación, enfoque en el logro, etc., y, las competencias gerenciales, que viene a ser el cúmulo de conocimientos, habilidades, conductas y actitudes que un individuo tiene para tener un resultado efectivo en una amplia gama de puestos y en diferentes tipos de organizaciones.

AGLALA ISNN 2215-7360

2014; 5 (1): 107-125 
Sin embargo, en la relación de inteligencia y la competencia, existen estudios como las de McClelland (1973), que argumenta que la inteligencia, a través de las mediciones del coeficiente intelectual, no da una correlación entre éxito e inteligencia, que permitiría predecir el éxito laboral y en la vida en general. Ahora, otros autores, como Leboyer (1997) refutan lo anterior, al expresar que se han dado varios estudios donde se demuestra que la medición de la inteligencia sí permite predecir altos niveles de desempeño en determinadas labores. Por lo tanto, es evidente que, aunque en ciertos casos, la inteligencia puede ser un factor importante para el éxito laboral, no es una regla inconmovible, debido a que existen personas que pueden tener altos niveles de coeficiente intelectual, pero no ser competentes, además de carecer de actitudes, como por ejemplo el trabajo en equipo, que no lo hace apto para el ejercicio de un determinado puesto de liderazgo o dirigencial.

Otra clasificación de competencias la da Gallego (2000), quien las divide en Diferenciadoras y de Umbral. Las primeras son características del individuo que le dan un desempeño sobresaliente, esto da a entender que son propias de cada persona, ya que pueden haber dos individuos bajo las mismas circunstancias laborales, pero una de ellas, actuar de manera superior. Las segundas, que permiten que pueda haber un desenvolvimiento normal y que han sido parte del enfoque tradicional de la selección de personal, es decir, buscar a la persona que pueda desenvolverse de manera adecuada en un cargo y que no necesariamente lo ejecute de manera sobresaliente. Ante esto, un aspecto importante que puede llevar a una persona tener una distinción sobresaliente ante otros en un área laboral, está relacionado con sus valores, como por ejemplo, el dar la milla extra, es decir, buscar siempre dar más para lo cual fue contratado. Este punto tiene mucho que ver con la actitud, la cual es la forma de reaccionar de un individuo ante una situación, lo cual va más allá de las aptitudes o preparación intelectual. AGLALA ISNN 2215-7360

2014; 5 (1): 107-125 
La planeación de los recursos humanos, permite situar la cantidad adecuada de individuos calificados en el puesto apropiado y el momento apropiado también. Además, Aramayo (2000), dice que la planeación de personal es la que permite ajustar la oferta de los vacantes disponibles y de los que van a ser buscados o se han de contratar, con las vacantes que se espera pueda contar la empresa en un tiempo determinado.

Por otro lado, Román (2009), ve a la planificación de recursos humanos como una actividad que es propia de las empresas y que busca integrar los objetivos de los individuos con los objetivos de la empresa. Esta planeación tiene unos objetivos básicos que son:

- La búsqueda de la optimización de los recursos humanos de la organización.

- Contar con la planilla necesaria en el tiempo.

- El sistema de formación y promoción de las personas en la empresa de acuerdo con las futuras necesidades.

- Incentivar el factor humano corporativo.

- Mejorar el clima laboral.

- Contribuir a incrementar el beneficio de la organización.

Para lograr la optimización de los recursos humanos, se debe comenzar con un adecuado proceso de selección de personal. La selección de personal es un proceso por medio del cual las organizaciones toman la decisión de qué aspirantes a una labor determinada será el más apto para ocuparla y desempeñarla. Es decir, es un proceso de toma de decisiones sobre el ajustar a los candidatos a los puestos que oferta una empresa (Salgado y Moscoso, 2008). Ahora, para poder llevar adelante este proceso, es necesario emplear instrumentos que evalúen a los aspirantes y que permitan la toma de decisión sobre la persona más indicada al cargo y, es requisito fundamental que tal proceso se lleve a cabo por un profesional capacitado y que pueda AGLALA ISNN 2215-7360

2014; 5 (1): 107-125 
utilizar los instrumentos determinados. En la misma línea, la selección de los recursos humanos es para Alles (2000), la "actividad de clasificación donde se escoge a aquello que tengan mayor probabilidad de adaptarse al cargo ofrecido para satisfacer las necesidades de la organización y del perfil (p. 115).

Entonces, los especialistas que llevarán a cabo el proceso de reclutamiento y selección deben de tener claridad sobre el puesto, las actividades y características que deben de tener las personas que ocuparán un cargo y que puedan desempeñarse efectivamente. El análisis del puesto y del perfil es el lente que permitirá visualizar las áreas de los candidatos y que tengan relación con el desempeño de una labor particular. Además, se debe tener presente que debe de existir equilibrio entre lo que se requiere y lo real que existe en el mercado.

\section{Servicio al cliente y competencias laborales en empresas de servicios}

El servicio al cliente es el resultado de un proceso por medio del cual cada área conoce su función de manera específica, por lo que es un diseño que conceptualiza las competencias especializadas que deben de ejecutar los trabajadores, desde el nivel directivo hasta el nivel operativo. La administración reconoce, según Daft (2007), que para que una organización empresarial tenga éxito tiene que tener en cuenta aspectos como son: el buen clima laboral, que los trabajadores tengan oportunidad para el crecimiento, cumplir las leyes laborales, etc., Ante esto, es notorio la importancia que estas variables tienen al momento del desempeño laboral por parte de los trabajadores y que repercutirán en el servicio que se brinde al cliente. Más aún, en momentos cruciales en lo que se requiera cubrir una necesidad o conseguir un propósito específico, como lo puede ser el resolver de la mejor manera posible los requerimientos de los 
clientes para lograr su satisfacción y, para que esto se pueda dar, es imperativo poner en obra las competencias para poder atender de manera efectiva al cliente.

Se decidió tomar como ejemplo a las empresas de servicio del sector turístico, en la forma en cómo se presentan las competencias en este tipo de ámbito empresarial. En este sector, por su amplia y diversificación de actividades productivas que ofertan y solicitadas por los usuarios, la cadena de servicio que componen a esta industria se da a través de las agencias de promoción y ventas, transporte, restaurantes, hoteles, sitios de interés turístico, tiendas de artesanías.

La dinámica del sector turístico, necesita un constante replanteamiento de las funciones que deben desempeñar cada uno de los empleados para poder enfrentar con éxito los permanentes y novedosos retos. El trabajo que se realiza en esta área empresarial es un $70 \%$ práctico, cara a cara con el cliente, debido a que el trabajador trabaja en áreas de servicio directo como son los aeropuertos, agencias de viajes, restaurantes, hoteles, parques diversos. En Colombia, las personas que prestan este servicio son en mayoría empíricos, tal como lo expresa Cabarcos (2006), contrastando con los países más desarrollados, los cuales cuentan con personal con mayor preparación intelectual.

La calidad del servicio que se brinda a los clientes es el elemento productivo y comercial que se constituye como un verdadero factor diferenciador y de mayor valor agregado. Esto debido a que el sentido de servicio que va de la mano con un producto requiere del desarrollo de mejores competencias especializadas por parte de los trabajadores que forman parte de la cadena del servicio. En el turismo, el trabajador tiene la oportunidad de ofrecer una experiencia única al cliente que vaya más allá del elemento transaccional, por medio de crear una relación, y esto de una satisfacción óptima del cliente.

AGLALA ISNN 2215-7360

2014; 5 (1): 107-125 
Por lo tanto, las competencias laborales al servir al cliente deben de ser entendida como una estrategia en la que todos los niveles de la empresa estén directa o indirectamente comprometidas con los clientes. Esto se dará si todos los procesos se encaminan al logro de los resultados misionales y a que los individuos que forman parte de la empresa interioricen una vocación legítima hacia el servicio y se comprometan de manera directa con los resultados de la organización. Además, al momento de seleccionar al personal idóneo a un puesto en una empresa de servicio, se debe de tener en cuenta atributos personales que son elementos de personalidad que se presentan a través del respeto, la adaptabilidad, la recursividad, destreza y sensibilidad social, persuasión, habilidades de comunicación, etc.

El aprendizaje, desarrollo y fortalecimiento de las competencias en un aspecto clave y crítico dentro de la cultura del servicio. Esto demanda la capacitación de manera continua de todos los trabajadores, poniendo énfasis en los que tienen trato directo con el cliente, pues según su desempeño dependerá la satisfacción y la fidelización del cliente. Todo lo anterior, aunado a la motivación, disposición y entrenamiento del trabajador.

El proceso de selección de personal en las organizaciones de servicio tiene que considerar el entorno que rodea a las empresas en sus tendencias mundiales, su impacto en los diferentes tipos de organizaciones y efecto en el proceso de selección de personal. Además, el proceso debe de insertarse en un escenario real, con políticas, leyes laborales y normas vigentes de cada país, y lo más importante, es un proceso de doble vía que implica el desempeño de roles de asesoramiento y orientación, demandantes de responsabilidades concretas del seleccionador hacia sus clientes.

Tomando en cuenta lo concerniente a los roles de asesoramiento y orientación, la selección de personal por competencias se revalora como un proceso que busca el AGLALA ISNN 2215-7360

2014; 5 (1): 107-125 
establecimiento de vínculos perdurables de mutuo beneficio para el individuo y la empresa, en cuanto a desarrollo personal y organizacional.

\section{La selección por competencias}

La selección por competencias se refiere a una técnica que los expertos en recursos humanos utilizan para seleccionar personal cuando su objetivo es encontrar profesionales que, además de una formación y experiencia adecuadas, posean unas competencias concretas predeterminadas por la empresa que ofrece el empleo.

El deber de las empresas de servicios es justamente prestar con eficiencia y responsabilidad sus operaciones a sus clientes, debido a ello, en el momento en que se presenta una vacante, la empresa analiza a sus mejores trabajadores de cada división o departamento, elabora la caracterización de las competencias que poseen, las cuales llegan a convertirse en estándar. De esta manera, según Laboris.net (s/f), al momento de seleccionar un trabajador se indagan perfiles que tengan similitud al de los individuos que tienen mejor rendimiento en la organización. Si la vacante es nueva, se toma como referente el listado base y se agregan las competencias concretas que demande cada situación.

Volviendo con Salgado y Moscoso (2008), estos autores hablan del modelo de selección de personal estratégico, el cual se fundamenta en una visión diferente de lo que se presenta en el mercado económico y laboral. Este modelo explica que la economía se desenvuelve en un escenario donde prima la volatilidad, debido a los ambientes económicos cambiantes. Esto seguido del proceso global que se manifiesta en la reducción de las fronteras. Ahora, este tipo de escenario demanda recursos humanos que trabajen en equipo y que se dé mayor interdependencia entre los empleados, seguido de la capacidad de aprendizaje, la innovación y AGLALA ISNN 2215-7360

2014; 5 (1): 107-125 
la creatividad, automotivación, enfoque del trabajo al servicio del cliente, la calidad, la virtualidad, estabilidad emocional, habilidades comunicacionales, etc.

Al llevarse a cabo la selección en la organización, lo que se busca es enlazar el mejor talento humano y que cumpla con el perfil que la empresa necesita, por lo que es necesario que los aspirantes cuenten con las formación académica correspondiente, cuenten con la experiencia adecuada a las funciones a ejecutar y se les analicen las competencias que tienen.

Es muy complicado el conocer a una persona a simple vista en su ser y en su comportamiento, debido a ello es que al momento de hacer la selección se debe de utilizar las herramientas necesarias que darán la información sobre los conocimientos, las competencias y motivaciones de los candidatos, lo cual, al fin y al cabo, permitirá hacer una selección por competencias. Aun así, la mente humana todavía es un enigma y por lo tanto, tal selección no será nunca cien por ciento perfecta. Con esto, lo que se busca es ser lo más certero posible en las predicciones sobre el desenvolvimiento laboral futuro en un puesto de trabajo y contratar al candidato competente para tal trabajo.

La empresa Ericsson presentó un modelo de competencias que fue ideado por Reus en el año 1997 (citado en Hoyos, 2010), el cual agrupa tres tipos de competencias para el reclutamiento y selección del talento humano que son: Primero, competencias de direccionamiento, que sirven para la puesta en marcha de los objetivos organizacionales, los planes estratégicos que tengan relación con la misión y visión organizacional, las cuales se pueden observar a través del liderazgo, comunicación gerencial, poder de decisión, delegación y seguimiento. Segundo, competencias técnicas y profesionales, que son las habilidades, características y conocimientos de forma específica para laborar de forma adecuada y eficiente y se observa a través de la planeación y control, rapidez, oportunidad y respuesta, análisis de la AGLALA ISNN 2215-7360

2014; 5 (1): 107-125 
información, comunicación oral y escrita y el seguimiento a las instrucciones dadas. Tercero, competencias humanas y de interacción, que permiten al trabajador interactuar de manera adecuada con otros individuos, sean dentro o fuera de la organización, los cuales se pueden observar a través de la responsabilidad, solución de conflictos, tolerancia, confianza, negociación, proactividad, relaciones interpersonales.

Lo anterior, permite promover y desarrollar cualidades que den diferencia ante otras compañías y es un gran aliado para el éxito de todas las actividades y den una orientación hacia el desarrollo de una ventaja competitiva que se traduce en el capital humano. Además, que genere comportamientos que lleven a mejorar el desempeño de las labores que sean asignadas a cada individuo dentro de la organización y, por ende, en una empresa de servicios. De esta manera, el talento humano repercute e influye en la competitividad corporativa, debido a que se ha alineado al direccionamiento estratégico y a la gerencia de procesos.

Según Sánchez (2013), el proceso de selección por competencias se presenta en varias fases a detallar:

\section{Preselección de candidatos}

En este punto ya se comienzan a tomar decisiones sobre si calza el perfil de competencias del aspirante al cargo. Por medio de la debida lectura y análisis de la hoja de vida los candidatos pueden ser clasificados en: aptos, que son individuos potenciales ante los requerimientos para el puesto; dudosos, ante los cuales hay falta de información se necesitaría una entrevista y se les puede tener en reserva; y rechazados, no cumplen el proceso para ser tomados por la organización. Además, se hace las respectivas verificaciones de datos y referencias como son los académicos, datos laborales y referencias personales.

AGLALA ISNN 2215-7360

2014; 5 (1): 107-125 


\section{Entrevista preliminar}

Ante las dudas que se puedan presentar en la preselección, es necesario llevar a cabo una entrevista con el fin de conocer las motivaciones e intereses reales de los aspirantes que formarán parte de la organización.

La entrevista se centra sobre acciones concretas que son expuestos por el entrevistado para poder encontrar hallazgos de comportamientos en el pasado del candidato que serán tomados en cuenta para predecir el desempeño ante un cargo. Se hacen preguntas conocidas como incidentes críticos o eventos conductuales.

\section{Pruebas}

Es muy utilizada por las empresas para seleccionar personal. Ahora, producto del enfoque en las competencias surge la entrevista focalizada o por competencias.

Se aplican las pruebas psicométricas y psicotécnicas. Las primeras, buscan la medición objetiva y estandarizada de una muestra del comportamiento y que buscan verificar la aptitud para prever y generalizar cómo se manifestará tal comportamiento en una labor determinada. Miden capacidades, intereses o aptitudes, la inteligencia, comprensión y fluidez verbal, intereses ocupacionales, actitudes, etc.

Los test se clasifican en:

- Test de inteligencia, que buscar ver la capacidad de aprender y resolver problemas

- Test de aptitudes, que busca evaluar las capacidades y aptitudes que sean necesarias para llevar a cabo una labor concreta.

AGLALA ISNN 2215-7360

2014; 5 (1): 107-125 
- Test de personalidad, que busca evaluar el carácter y temperamento existente en el individuo, que son consecuencia de procesos biológicos, psicológicos y sociales. Estos rasgos en la persona son de más difícil variación o modificación.

- Test proyectivos, el cual busca evaluar el carácter del candidato. Esto se da a través de la presentación de estímulos no estructurados, el cual, una vez realizados, dan una proyección de lo que hay en el interior del individuo.

Las segundas, buscan evaluar habilidades concretas que son necesarias para desempeñar un puesto laboral. Esta técnica busca observar los comportamientos en la práctica de un individuo cuando esté ejecutando una labor y para ello es necesario contar con protocolos de observación por medio de los cuales los evaluadores sistematizan la información acumulada durante el proceso de observación, sea dentro o fuera del ámbito laboral. Las pruebas usadas en este punto son las Pruebas Situaciones, que son técnicas y ejercicios que permiten simular, de manera parcial o total, una situación determinada en la que los postulantes deben de poner de manifiesto las competencias que demanda, de manera eficaz, de una actividad laboral. Se emplearán la técnica de análisis de caso escrito, la técnica de evaluación de competencias, juego de roles y la técnica de presentación oral y escrita.

\section{Informe de selección}

Para llevar a cabo este proceso, se debe de codificar en un informe de selección toda la información recabada de cada uno de los candidatos. Esto permite elegir al más competente de entre todos los candidatos para el cargo. En el informe se deben de caracterizar de manera fundamental: identificación del candidato, su formación, su experiencia, sus intereses, su disponibilidad, sus motivaciones y que resultados arrojó el candidato en las pruebas y/o AGLALA ISNN 2215-7360

2014; 5 (1): 107-125 
entrevistas a nivel de las competencias y, como punto final, dar una valuación general que justifique sobre si es o no ideal para el cargo. El profesional responsable de la selección organiza de manera jerarquizada a los candidatos, según lo que se requiera en el puesto y en la empresa.

\section{Toma de decisión - elección}

El responsable de la selección del futuro trabajador debe de analizar el grado de concordancia de tres factores, como son la persona, el puesto y la organización. El que más parece cumplir con todo el proceso será el seleccionado y luego contratado. Una vez que el trabajador esté en su campo laboral se le hará un seguimiento para ver si está produciéndose la adaptación esperada.

Se puede recalcar que el proceso de selección de personal por competencias se diferencia de un proceso de selección tradicional por la metodología empleada, no necesariamente por los pasos. Los métodos se fundamentan en la identificación y evaluación de las competencias.

Para evaluar las competencias se recurre a nuevos instrumentos psicológicos que deben de ser concebidos con base a una interpretación dinámica que se asemeja a las competencias. Ahora, el éxito del proceso de selección por competencias se fundamenta en la adecuada definición de las competencias organizacionales y funcionales, que deben de estar ligadas a la planeación estratégica de la organización. Esto es importante al momento de determinar las técnicas y los métodos más eficientes para la identificación y evaluación de las competencias en los nuevos y actuales empleados.

Las pruebas situaciones en comparación a las pruebas tradicionales, elevan la validez y la capacidad predictiva, debido a que enfrenta al trabajador ante una situación más próxima a su

AGLALA ISNN 2215-7360

2014; 5 (1): 107-125 
realidad profesional, lo cual contribuye a conseguir un proceso más objetivo al evaluar los comportamientos.

\section{Conclusiones}

La selección de personal por competencias constituye una herramienta indispensable para las empresas de servicios como una fórmula para ganar flexibilidad en la empresa y optimizar los procesos. Su aplicación produce cambios profundos en la forma de concebir el desempeño, la evaluación, la retribución y el desarrollo de carrera. Pone de relieve que las conductas son importantes para alcanzar los resultados, a diferencia de la tradicional tendencia que busca controlar solo el resultado. Debido a esto, resulta una guía para la persona que se integre a un puesto, logrando disminuir la incertidumbre que genera no saber qué es lo que se espera de su trabajo y más bien incentivar el mejoramiento continuo del empleado. Sin embargo, cada organización deberá plantear sus necesidades y en base a ello, determinar las que deberá usar, debido a que las necesidades, filosofía y estructura organizacional son variables dependiendo de la empresa. Es más, aunque se logren seleccionar los mejores candidatos, esto no asegura el logro total de los objetivos de la empresa, por lo que es necesario motivar constantemente a los trabajadores mediante reconocimientos verbales o económicos. Además, se deberá considerar que si las personas ocupan puestos multifuncionales, su remuneración debe ser proporcional a las tareas y responsabilidades a su cargo.

\section{Referencias bibliográficas}

Aramayo, R. (2000). Planificación Estratégica de los Recursos Humanos. México: Cecsa. Alles, M. (2000). Dirección estratégica de recursos humanos. Gestión por competencias. AGLALA ISNN 2215-7360

2014; 5 (1): 107-125 
Barcelona: Granica.

Blanco, A. (2007). Trabajadores competentes. Introducción y reflexiones sobre la gestión de recursos humanos por competencias. Madrid: EsicEditoral.

Cabarcos, N. (2006). Promoción y venta de servicios turísticos. Madrid: Editorial Ideas Propias.

Daft, R. (2007). Teoría y diseño organizacional. México: Cencage Learning Editores.

Gallego, M. (2000). Gestión humana basada en competencias. Recuperado el 16 de agosto de 2014 de http://publicaciones.eafit.edu.co/index.php/revista-universidadeafit/article/view/1026/926

Helleriegel, D. (2009). Administración un enfoque basado en competencias. México: CenageLearning.

Hoyos, W. (2010). Diseño de perfil del cargo. Revista Universidad EAFIT, 46, 11-30.

Laboris.net (2008). Selección por competencias. Recuperado el 05 de agosto de 2014 de http://www.laboris.net/static/em_diccionario_seleccion-competencias.aspx

Leboyer, L. (1997). Gestión de las competencias. Cómo analizarlas, cómo evaluarlas, como desarrollarlas. Barcelona: Gestión 2000.

McClelland, D. (1973). Testing for competence rather than intelligence. The International Journal of Public Sector Management. 23, 6, 275-285.

Montoya, J. (2010). Conceptos claves sobre competencia. Recuperado el 20 de agosto de 2014 de http://acreditacion.unillanos.edu.co/contenidos/JORGE\%20MONTOYA/2\%BA\%20ENC UENTRO\%20SEPT\%2027\%20Y\%2028/Conceptosclaves_competbencias.pdf Pereda, S. y Berrocal, F. (2011). Dirección y gestión de recursos humanos por competencias. Madrid: Centro de Estudios Ramón Areces.

AGLALA ISNN 2215-7360

2014; 5 (1): 107-125 
Román, Y. (2009). Cartilla de gestión humana. Colombia: Institución Universitaria CEIPA. Real Academia de la Lengua. (2014). Real Academia Española. Recuperado el 15 de agosto de 2014 de http://lema.rae.es/drae/?val=competencia

Salgado, J., y Moscoso, S. (2008). Selección de personal en la empresa y las AAPP: de la visión tradicional a la visión estratégica. Revista del consejo de colegios oficiales de psicólogos, 29, 16-24.

Sánchez, C. (2013). Selección de recursos humanos por competencias. Tesis de pregrado. Universidad de Valladolid, Valladolid, España.

AGLALA ISNN 2215-7360

2014; 5 (1): 107-125 